Letters to the Editor

\title{
Severe Sepsis Due to Raoultella Planticola
}

\author{
Chiara Casarsa, Filippo Mearelli and Gianni Biolo \\ Department of Medical, Unit of Clinica Medica Generale e Terapia Medica \\ University of Trieste, Strada di Fiume, 44734149 Cattinara Trieste, Italy
}

\author{
Article history \\ Received: 28-03-2017 \\ Revised: 31-03-2017 \\ Accepted: 04-04-2017 \\ Corresponding Author: \\ Chiara Casarsa \\ Department of Medical, Unit of \\ Clinica Medica Generale e \\ Terapia Medica University of \\ Trieste, Strada di Fiume, 447 \\ 34149 Cattinara Trieste, Italy \\ E-mail: chiaracasarsa@gmail.com
}

\begin{abstract}
Raultella planticola usually invaded immune compromised patients and has been considered a relatively harmless Gram-negative bacteria. We reported a case of a women with a new pancreatic cancer diagnosis that abruptly develop severe sepsis. A Raoultella planticola was yielded in blood cultures. This case suggest Raoultella as pathogen causing life threatening community acquired infection.
\end{abstract}

Keywords: Raoultella Planticola, Infection, Cholangitis, Severe Sepsis

\section{Introduction}

Severe sepsis due to Raoultella planticola is a quite rare and usually nosocomial acquired infection.

We hereby report an illustrative case of bacteremic life-threatening infection due to community-acquired Raoultella planticola.

\section{Case Report}

In April 2015 a 55-year-old woman visited our hospital with asymptomatic jaundice.

She had a past medical history significant for Sjogren's syndrome and iodine contrast allergy.

She denied being on any regular medications.

After $24 \mathrm{~h}$ the clinical status changed: On clinical examination the patient satisfied two criteria for systemic inflammatory response syndrome (BT $39^{\circ}$ and tachycardia of $110 \mathrm{bpm}$ ) and appeared confused.

The performed abdominal examination did not show any significant abnormality; neither rigor nor neurological defect was detected.

Laboratory test showed an increased white cell count $\left(2,4 \times 10^{3} / \mathrm{microL}\right), \quad$-reactive protein $150 \mathrm{mg} \mathrm{L}^{-1}$, bilirubin $10 \mathrm{mg} \mathrm{dL}^{-1}$, alkaline phosphatase $180 \mathrm{U} \mathrm{L}^{-1}$, alanine amino transferase $58 \mathrm{U} \mathrm{L}^{-1}$, gamma-glutamyl transferase $140 \mathrm{U} \mathrm{L}^{-1}$.

The abdominal ultrasonography showed a pancreatic lesion and dilated intrahepatic ducts; a biliary stent was inserted and the CT-PET confirmed the solid lesions with a suspect lymph node at the hilum (low standardized uptake values).
Surgical specimen confirmed the diagnosis of pancreatic mucinous adenocarcinoma.

About $48 \mathrm{~h}$ after admission, two blood culture specimens with drown before procedures yielded a sensitive to every antibiotic Raoultella planticola. Urine remained negative. The patient was empirically treated with cefriaxone $1 \mathrm{gr} / \mathrm{die}$ in the suspect of cholangitis; after susceptibility testing, antimicrobial therapy was deescalated for 14 days with good clinical response.

\section{Discussion}

R. planticola is a Gram-negative anaerobic bacillus belonging to the Enterobacteriacee family; it was previously described as Klebsiella planticola, but in 2001 was reclassified as a new genus.

Members of the Raoultella genus include Raoultella electrica, R. terrigena, R. planticola and R. ornithinolytica.

$\mathrm{R}$. planticola has been reported as clinical isolates in human sputum, stools, wounds and urine, but it is a rare cause of invasive human infection (Teo et al., 2012).

A complication in identifying R. planticola in humans is that it is difficult to isolate the pathogen in culture and that it can be easily mistaken for other genera within the family Enterobacteriacea (Lam and Salit, 2014).

The infection is frequently associated with a history of invasive procedures and an immune compromised status. The major underlying disease has been found to be malignancy.

The most common primary sites of infection are the biliary and urinary tracts (Teo et al., 2012).

The percentage of bacteremia reported in literature varies from $0.3 \%$ (as described in a recent review occurring over a 4-year period) (Chun et al., 2014) to 
$9.4 \%$ of cases, as reported in a recent analysis over a 5year period (Boattini et al., 2016).

Most strains showed susceptibility to cephalosporin, as well as to carbapenem, although nosocomial emergence of Carbapenem-Resistant R. Planticola (CRRP) has recently been highlighted (UTI in females). No resistance to fluoroquinolone agents has been observed (Chun et al., 2014).

The majority of patients survive the infection: Since its identification in 1984 only 17 patients with severe sepsis have been reported. Among these cases only one was, like ours, community-acquired, while the others were nosocomial (Ershadi et al., 2014).

In the cases where the patient did not survive, either blood cultures revealed evidence of polymicrobial infection or Raoultella planticola was found to be multidrug-resistant, due to its carrying the carbapenemresistent bla KPC gene (Ershadi et al., 2014).

Given the difficulty in isolating the described pathogen in culture, the real incidence of this life-threatening infection is surely underestimated. The above-mentioned case is, in fact, the second reported in literature.

\section{Conclusion}

Clinicians should be aware that Raoultella planticola has the potential to cause severe infections, even when community-acquired.

\section{Author's Contributions}

Chiara Casarsa: Contributed to the writing.

Filippo Mearelli: Contributed to the writing and the reviewing.

Gianni Biolo: Contributed to the reviewing and editing.

\section{Ethics}

This article is original and contains unpublished material. The corresponding author confirms that all of the other authors have read and approved the manuscript and no ethical issues involved.

\section{References}

Teo, I., J. Wild, S. Ray and D. Chadwick, 2012. A rare case of cholecystitis caused by raoultella planticola. Case Reports Med. DOI: 10.1155/2012/601641

Lam, P.W. and I.E. Salit, 2014. Raoultella planticola bacteriemia following consumption of seafood. Can J. Infect. Dis. Med. Microbiol., 25: e83-e84.

Chun, S., Y.W. Yun, H.J. Huh and N.Y. Lee, 2014. Low virulence? Clinical characteristics of raoultella planticola bacteremia. Infection, 42: 899-904.

Boattini, M., A. Almeida, C. Cardoso, C. Machado and Z. Vesza et al., 2016. Infections on the rise: Raoultella spp., clinical and microbiological findings from a retrospective study, 2010-2014. Infect. Dis. (Lond), 48: 87-91

Ershadi, A., E. Weiss, E. Verduzco, D. Chia and M. Sadigh, 2014. Emerging pathogen: A case and review of raoultella planticola. Infection, 42: 1043-46. 